[3] M. Mateos, Problemas de control óptimo gobernados por ecuaciones semilineales con restricciones de tipo integral sobre el gradiente del estado. Ph. D. Thesis, University of Cantabria, 2000.

Acknowledgements: The author has been partially supported by D.G.I. (Spain), Grant BFM2002-02488.

\title{
3 Remarks on the controllability of some parabolic equations and systems
}

\author{
Enrique Fernández Cara
}

Dpto. E.D.A.N, Universidad de Sevilla

cara@us.es

\begin{abstract}
We present a review of recent results concerning the null controllability of parabolic systems. Among others, we will consider the heat equation, the Burgers, Navier-Stokes and Ginzburg-Landau equations, etc. We will also indicate some open questions.
\end{abstract}

\subsection{Introduction. Controllability and observability}

Let us first recall some general ideas that apply to a large family of (linear and nonlinear) evolution problems.

Suppose that we are considering a state equation of the form

$$
\left\{\begin{array}{l}
y_{t}-A(y)=B v, \quad t \in(0, T) \\
y(0)=y^{0}
\end{array}\right.
$$

which governs the behavior of a physical system. It is assumed that $A: D(A) \subset$ $H \mapsto H$ is a (generally nonlinear) operator, $y:[0, T] \mapsto H$ is the state, i.e. the variable that serves to identify the physical properties, $v:[0, T] \mapsto U$ is the control, i.e. the variable we can choose, $B \in \mathcal{L}(U ; H)$ and $y^{0} \in H(U$ and $H$ are Hilbert spaces).

Suppose that (3.1) is well-posed, in the sense that for each $y^{0} \in H$ and each $v \in L^{2}(0, T ; U)$, (3.1) possesses exactly one solution. Then the null controllability problem for (3.1) can be stated as follows:

For each $y^{0} \in H$, find $v \in L^{2}(0, T ; U)$ such that the corresponding solution of (3.1) satisfies $y(T)=0$.

For each system of the form (3.1), the null controllability problem leads to several interesting questions. Among them, let us mention the following:

- First, are there controls $v$ such that $y(T)=0$ ? 
- Then, if this is the case, which is the cost we have to pay to drive $y$ to zero? In other words, which is the minimal $L^{2}(0, T ; U)$-norm of a control $v$ satisfying this property?

- How can these controls be computed?

The controllability of differential systems is a very relevant area of research and has been the subject of many papers the last years. In particular, the null controllability of linear PDEs was first analyzed in [25, 26, 22, 23, 18, 21]. For semilinear systems, the first contributions have been given in $[27,6,14]$.

In this paper, we will try to recall some known results concerning among others the heat equation, the Burgers, Navier-Stokes and Ginzburg-Landau equations, etc. We will also indicate some open questions.

\subsection{The classical heat equation. Carleman estimates}

In this Section, we will consider the controlled heat equation, complemented with initial and Dirichlet boundary conditions:

$$
\begin{cases}y_{t}-\Delta y=v 1_{\omega}, & (x, t) \in \Omega \times(0, T), \\ y=0, & (x, t) \in \partial \Omega \times(0, T), \\ y(0)=y^{0}, & x \in \Omega .\end{cases}
$$

Here, $\Omega \subset \mathbb{R}^{N}$ is a nonempty bounded open set, $\omega \subset \subset \Omega$ is a (small) nonempty open subset $\left(1_{\omega}\right.$ is the characteristic function of $\omega$ ) and $y^{0} \in L^{2}(\Omega)$. It is well known that, for every $y^{0} \in L^{2}(\Omega)$ and every $v \in L^{2}(\omega \times(0, T))$, there exists a unique solution $y$ to $(3.2)$, with $y \in L^{2}\left(0, T ; H_{0}^{1}(\Omega)\right) \cap C^{0}\left([0, T] ; L^{2}(\Omega)\right)$.

In this context, the null controllability problem reads:

For each $y^{0} \in L^{2}(\Omega)$, find $v \in L^{2}(\omega \times(0, T))$ such that the corresponding solution of (3.2) satisfies

$$
y(T)=0 \text { in } \Omega .
$$

Together with (3.2), for each $\varphi^{1} \in L^{2}(\Omega)$, we can introduce the associated adjoint system

$$
\begin{cases}-\varphi_{t}-\Delta \varphi=0, & (x, t) \in \Omega \times(0, T), \\ \varphi=0, & (x, t) \in \partial \Omega \times(0, T), \\ \varphi(T)=\varphi^{1}, & x \in \Omega .\end{cases}
$$

Then, it is well known that the null controllability of (3.2) is in practice equivalent to the following property:

There exists $C>0$ such that

$$
\|\varphi(0)\|_{L^{2}}^{2} \leq C \iint_{\omega \times(0, T)}|\varphi|^{2} d x d t \quad \forall \varphi^{1} \in L^{2}(\Omega) .
$$


This is called the observability of (3.4); it is said that (3.5) is an observability estimate. We thus find that, in order to solve the null controllability problem for (3.2), it suffices to prove (3.5).

The estimates (3.5) are a consequence of the so called global Carleman inequalities. These have been introduced in the context of the controllability of PDEs by Fursikov and Imanuvilov, see $[18,14]$. When they are applied to the solutions of the adjoint systems (3.4), they take the form

$$
\iint_{\Omega \times(0, T)} \rho^{2}|\varphi|^{2} d x d t \leq K \iint_{\omega \times(0, T)} \rho^{2}|\varphi|^{2} d x d t \quad \forall \varphi^{1} \in L^{2}(\Omega),
$$

where $\rho=\rho(x, t)$ is an appropriate weight, depending on $\Omega, \omega$ and $T$ and the constant $K$ only depends on $\Omega$ and $\omega$. In order to prove (3.6), we have to use a weight $\rho$ decreasing exponentially to zero as $t \rightarrow 0$ and $t \rightarrow T$.

Combining (3.6) and the dissipativity of the backwards heat equation (3.4), it is not difficult to deduce (3.5) for some $C$ only depending on $\Omega, \omega$ and $T$.

As a consequence, we have:

Theorem 3.1 The linear system (3.2) is null controllable. In other words, for each $y^{0} \in L^{2}(\Omega)$, there exists $v \in L^{2}(\omega \times(0, T))$ such that the corresponding solution of (3.2) satisfies (3.3).

There are many generalizations and variants of this result that provide the null controllability of other linear PDEs:

- Time-space dependent (sufficiently regular) coefficients can be included, other boundary conditions can be used to complement the PDE, boundary control (instead of distributed control) can be imposed, etc. For a review of recent applications of Carleman inequalities to the controllability of parabolic systems, see [10].

- The controllability of Stokes-like systems can also be analyzed with these techniques. This includes systems of the form

$$
y_{t}-\Delta y+(a \cdot \nabla) y+(y \cdot \nabla) b+\nabla p=v 1_{\omega}, \quad \nabla \cdot y=0,
$$

where $a$ and $b$ are regular enough; see for instance [11].

- Other linear parabolic (non-scalar) systems can also be considered, etc.

As mentioned above, an interesting question related to theorem 3.1 concerns the cost of null controllability. One has the following result from [13]:

Theorem 3.2 For each $y^{0} \in L^{2}(\Omega)$, let us set

$$
C\left(y^{0}\right)=\inf \left\{\|v\|_{L^{2}(\omega \times(0, T))} \text { : the solution of }(3.2) \text { satisfies } y(T)=0 \text { in } \Omega\right\} \text {. }
$$

Then we have the following estimate

$$
C\left(y^{0}\right) \leq \exp \left(C\left(1+\frac{1}{T}\right)\right)\left\|y^{0}\right\|_{L^{2}},
$$

where the constant $C$ only depends on $\Omega$ and $\omega$. 
Remark 3.1 Notice that theorem 3.1 ensures the null controllability of (3.2) for any $\omega$ and $T$. This is a consequence of the fact that, in a parabolic equation, the information is transmitted at infinite speed. For instance, this is not the case for the wave equation. For the latter, null controllability does not always hold. Contrarily, the couple $(\omega, T)$ has to satisfy appropriate geometrical assumptions; see [23] and [3] for more details.

\subsection{Positive and negative controllability results for the one- dimensional Burgers equation}

In this section, we will be concerned with the null controllability of the following system for the Burgers equation:

$$
\begin{cases}y_{t}-y_{x x}+y y_{x}=v 1_{\omega}, & (x, t) \in(0,1) \times(0, T), \\ y(0, t)=y(1, t)=0, & t \in(0, T), \\ y(x, 0)=y^{0}(x), & x \in(0,1) .\end{cases}
$$

Some controllability properties of (3.9) have been studied in [14] (see Chapter 1, Theorems 6.3 and 6.4). There, it is shown that one cannot reach (not even approximately) stationary solutions of (3.9) with large $L^{2}(0,1)$-norm at any time $T$. In other words, with the help of one control, the solutions of the Burgers equation cannot go anywhere at any time.

For each $y^{0} \in L^{2}(0,1)$, let us introduce

$$
T\left(y^{0}\right)=\inf \{T>0:(3.9) \text { is null controllable at time } T\} .
$$

Then, for each $r>0$, let us define the quantity

$$
T^{*}(r)=\sup \left\{T\left(y^{0}\right):\left\|y^{0}\right\|_{L^{2}} \leq r\right\} .
$$

Our main purpose is to show that $T^{*}(r)>0$, with an explicit sharp estimate in terms of $r$, which in particular implies that (global) null controllability at any positive time does not hold for (3.9).

More precisely, let us set $\phi(r)=\left(\log \frac{1}{r}\right)^{-1}$. We have the following result from [7]:

Theorem 3.3 One has

$$
C_{0} \phi(r) \leq T^{*}(r) \leq C_{1} \phi(r) \text { as } r \rightarrow 0
$$

for some positive constants $C_{0}$ and $C_{1}$ not depending of $r$.

Remark 3.2 The same estimates hold when the control $v$ acts on system (3.9) through the boundary only at $x=1$ (or only at $x=0$ ). When (3.9) is controlled at both points $x=0$ and $x=1$, it is unknown whether we still have an estimate from below for $T^{*}(r)$. 
Sketch of the proof of theorem 3.3: The proof of the estimate from above in (3.10) can be obtained by solving the null controllability problem for (3.9) via a (more or less) standard fixed point argument, using global Carleman inequalities to estimate the control and energy inequalities to estimate the state and being very careful with the role of $T$ in these inequalities.

More precisely, let us first consider the associated linear system

$$
\begin{cases}y_{t}-y_{x x}+a(x, t) y_{x}=v 1_{\omega} & (x, t) \in(0,1) \times(0, T), \\ y(0, t)=y(1, t)=0 & t \in(0, T) \\ y(x, 0)=y^{0}(x) & x \in(0,1)\end{cases}
$$

where $a \in L^{\infty}(Q)$ and $\|a\|_{\infty} \leq 1$.

There exist controls $v \in L^{\infty}(\omega \times(0, T))$ such that the associated states $y$ satisfy

$$
y(x, T)=0 \text { in }(0,1)
$$

and one has

$$
\|v\|_{\infty} \leq e^{K / T}\left\|y^{0}\right\|_{L^{2}}
$$

for some $K>0$ independent of $y^{0}$.

In order to prove this, it suffices to adapt the arguments in the previous section, paying special attention to the estimate of the observability constant.

This leads to a local controllability result for (3.9). More precisely, one has the following result, whose proof is sketched at the end of this section:

Lemma 3.4 Assume that the initial data satisfies

$$
\left\|y^{0}\right\|_{\infty} \leq \frac{1}{2} \quad \text { and } \quad\left\|y^{0}\right\|_{L^{2}} \leq \frac{1}{2 T} e^{-K / T}
$$

Then there exist controls $v$ such that the associated states in (3.9) satisfy (3.12).

Let us now finish the proof of the right inequality in (3.10). Let $r>0$ be given and let us introduce the set

$$
Z(r)=\left\{z:\|z\|_{\infty} \leq \frac{1}{2}, \quad\|z\|_{L^{2}} \leq r\right\}
$$

Starting from $y^{0} \in L^{2}(0,1)$ with $\left\|y^{0}\right\|_{L^{2}} \leq r$, we first let evolve the solution freely. From the parabolic regularity of the heat equation, we have that the solution of (3.9) satisfies

$$
\|y(\cdot, t)\|_{\infty} \leq C_{2} t^{-1 / 4}\left\|y^{0}\right\|_{L^{2}} \quad \forall t>0
$$

where $C_{2}$ is a constant (see for instance [20]). Accordingly, if we set $T_{0}=$ $\left(2 C_{2}\right)^{4} r^{4}$, we have $y\left(T_{0}\right) \in Z(r)$.

Then, starting at time $t=T_{0}$, we can apply to (3.9) a control $v$ such that the associated solution satisfies (3.12) at time $T=T_{0}+T_{1}$, where

$$
T_{1}=\frac{K}{\log (1 / r)} \text {. }
$$


Indeed, (3.14) is satisfied for $T=T_{1}$ (since $\left.r \leq\left(1 / 2 T_{1}\right) \exp \left\{-C^{*} / T_{1}\right\}\right)$. Consequently, from lemma 3.4 we see that such a control exists.

This proves that $T^{*}(r) \leq C_{1} \phi(r)$ for some $C_{1}$ as $r \rightarrow 0$.

Let us now turn to the proof of the estimate from below in (3.10), that is inspired by the arguments in [1].

We will prove that there exist positive constants $C_{0}$ and $C_{0}^{\prime}$ such that, for any sufficiently small $r>0$, we can find initial data $y^{0}$ satisfying $\left\|y^{0}\right\|_{L^{2}} \leq r$ with the following property: for any state $y$ associated to $y^{0}$, one has

$$
|y(x, t)| \geq C_{0}^{\prime} r \text { for some } x \in(0,1) \text { and any } t: 0<t<C_{0} \phi(r) .
$$

Let us set $T=\phi(r)$ and let $\rho_{0} \in(0,1)$ be such that $\left(0, \rho_{0}\right) \cap \omega=\emptyset$. We can suppose that $0<r<\rho_{0}$. Let us choose $y^{0} \in L^{2}(0,1)$ such that $y^{0}(x)=-r$ for all $x \in\left(0, \rho_{0}\right)$ and let us denote by $y$ an associated solution of (3.9).

Let us introduce the function $Z=Z(x, t)$, with

$$
Z(x, t)=\exp \left\{-\frac{2}{t}\left(1-e^{-\rho_{0}^{2}\left(\rho_{0}-x\right)^{3} /\left(\rho_{0} / 2-x\right)^{2}}\right)+\frac{1}{\rho_{0}-x}\right\} .
$$

Then one has $Z_{t}-Z_{x x}+Z Z_{x} \geq 0$.

Let us now set $w(x, t)=Z(x, t)-y(x, t)$. It is immediate that

$$
\begin{cases}w_{t}-w_{x x}+Z Z_{x}-y y_{x} \geq 0, & (x, t) \in\left(0, \rho_{0}\right) \times(0, T), \\ w(0, t) \geq 0, \quad w\left(\rho_{0}, t\right)=+\infty, & t \in(0, T), \\ w(x, 0)=r, & x \in\left(0, \rho_{0}\right)\end{cases}
$$

and, consequently, $w^{-}(x, t) \equiv 0$. Indeed, let us multiply the differential equation in (3.16) by $-w^{-}$and let us integrate in $\left(0, \rho_{0}\right)$. Since $w^{-}$vanishes at $x=0$ and $x=\rho_{0}$, after some manipulation we find that

$$
\begin{aligned}
& \frac{1}{2} \frac{d}{d t} \int_{0}^{\rho_{0}}\left|w^{-}\right|^{2} d x+\int_{0}^{\rho_{0}}\left|w_{x}^{-}\right|^{2} d x \\
& \quad=\int_{0}^{\rho_{0}} w^{-}\left(Z Z_{x}-y y_{x}\right) d x \leq C \int_{0}^{\rho_{0}}\left|w^{-}\right|^{2} d x .
\end{aligned}
$$

Hence,

$$
y \leq Z \quad \text { in }\left(0, \rho_{0}\right) \times(0, T) .
$$

Let us set $\rho_{1}=\rho_{0} / 2$ and let us introduce the solution $u$ of the auxiliary system

$$
\begin{cases}u_{t}-u_{x x}+u u_{x}=0, & (x, t) \in\left(0, \rho_{1}\right) \times(0, T), \\ u(0, t)=Z\left(\rho_{1}, t\right), \quad u\left(\rho_{1}, t\right)=Z\left(\rho_{1}, t\right), & t \in(0, T), \\ u(x, 0)=-\widetilde{r}(x), & x \in\left(0, \rho_{1}\right),\end{cases}
$$

where $\widetilde{r}$ is any regular function satisfying the following: $\widetilde{r}(0)=\widetilde{r}\left(\rho_{1}\right)=0$; $\widetilde{r}(x)=r$ for all $x \in\left(\delta \rho_{1},(1-\delta) \rho_{1}\right)$ and some $\delta \in(0,1 / 4) ;-r \leq-\widetilde{r}(x) \leq 0$;

$$
\left|\widetilde{r}_{x}\right| \leq C r \text { and }\left|\widetilde{r}_{x x}\right| \leq C \text { in }\left(0, \rho_{1}\right)
$$


where $C=C\left(\rho_{1}\right)$ is independent of $r$. Taking into account (3.18) and that $u_{x}, y \in L^{\infty}\left(\left(0, \rho_{1}\right) \times(0, T)\right)$ (see lemma 3.5 below), a standard application of Gronwall's lemma shows that

$$
y \leq u \text { in }\left(0, \rho_{1}\right) \times(0, T) .
$$

We will prove that, for some appropriate choices of $C_{0}$ and $C_{0}^{\prime}, u\left(\rho_{1} / 2, t\right)$ remains below $-C_{0}^{\prime} r$ for any time $t<C_{0} \phi(r)$. This, together with $(3.21)$, will prove theorem 3.3.

We will need the following lemma, whose proof can be found in [7]:

Lemma 3.5 One has

$$
|u| \leq C r \text { and }\left|u_{x}\right| \leq C r^{1 / 2} \quad \text { in }\left(0, \rho_{1}\right) \times(0, \phi(r)),
$$

where $C$ is independent of $r$.

A consequence of $(3.22)$ is that $u_{t}-u_{x x} \leq C^{*} r^{3 / 2}$ in $\left(0, \rho_{1}\right) \times(0, \phi(r))$ for some $C^{*}>0$. Let us consider the functions $p$ and $q$, given by $p(t)=C^{*} r^{3 / 2} t-r$ and $q(x, t)=c\left(e^{-\left(x-\left(\rho_{1} / 4\right)\right)^{2} / 4 t}+e^{-\left(x-3\left(\rho_{1} / 4\right)\right)^{2} / 4 t}\right)$. It is then clear that $b=u-p-q$ satisfies

$$
\begin{gathered}
b_{t}-b_{x x} \leq 0 \text { in }\left(\rho_{1} / 4,3 \rho_{1} / 4\right) \times(0, \phi(r)), \\
b\left(\rho_{1} / 4, t\right) \leq Z\left(\rho_{1}, t\right)-C^{*} r^{3 / 2} t+r-c\left(1+e^{-\rho_{1}^{2} /(16 t)}\right) \text { for } t \in(0, \phi(r)), \\
b\left(3 \rho_{1} / 4, t\right) \leq Z\left(\rho_{1}, t\right)-C^{*} r^{3 / 2} t+r-c\left(1+e^{-\rho_{1}^{2} /(16 t)}\right) \text { for } t \in(0, \phi(r)), \\
b(x, 0)=0 \text { for } x \in\left(\rho_{1} / 4,3 \rho_{1} / 4\right) .
\end{gathered}
$$

Obviously, in the definition of $q$, the constant $c$ can be chosen large enough to have $Z\left(\rho_{1}, t\right)-C^{*} r^{3 / 2} t+r-c\left(1+e^{-\rho_{1}^{2} /(16 t)}\right)<0$ for any $t \in(0, \phi(r))$. If this is the case, we get $u \leq p+q$ and, in particular,

$$
u\left(\rho_{1} / 2, t\right) \leq(p+q)\left(\rho_{1} / 2, t\right)=2 c e^{-\rho_{1}^{2} /(64 t)}+C^{*} r^{3 / 2} t-r .
$$

Therefore, we see that there exist $C_{0}$ and $C_{0}^{\prime}$ such that $u\left(\rho_{1} / 2, t\right)<-C_{0}^{\prime} r$ for any $t \in\left(0, C_{0} \phi(r)\right)$.

This proves (3.10) and, consequently, ends the proof of theorem 3.3.

Sketch of the proof of lemma 3.4: The proof of this lemma is standard but will be given here for completeness.

Let us introduce the fixed-point mapping $\mathcal{A}: L^{2}(Q) \longmapsto L^{2}(Q)$ given by

$$
\mathcal{A}(z)=y
$$

where $y$ is the solution of

$$
\begin{cases}y_{t}-y_{x x}+z(x, t) y_{x}=v 1_{\omega} & (x, t) \in(0,1) \times(0, T), \\ y(0, t)=y(1, t)=0 & t \in(0, T) \\ y(x, 0)=y^{0}(x) & x \in(0,1)\end{cases}
$$


for a control $v$ such that (3.13) holds and $y(T)=0$ in $\Omega$.

Let $K:=\left\{z \in L^{2}(Q):\|z\|_{\infty} \leq 1\right\}$, which is a convex and closed set in $L^{2}(Q)$. We are going to see that $\mathcal{A}$ fulfills the hypothesis of Kakutani's fixedpoint theorem for this $K$.

- An application of the classical maximum principle yields

$$
\|y\|_{\infty} \leq T\|v\|_{\infty}+\left\|y^{0}\right\|_{\infty} .
$$

Now, from (3.13) and conditions (3.14), we deduce

$$
\|y\|_{\infty} \leq \frac{1}{2}+\frac{1}{2}=1
$$

Consequently, $\mathcal{A}$ maps $K$ into $K$.

- For any $z \in K, \mathcal{A}(z)$ is a closed and convex subset of $L^{2}(Q)$. This is very easy to check so the proof is left to the reader.

- Let us finally prove that $\mathcal{A}$ is upper semicontinuous in $L^{2}(Q)$. Let $\left\{z_{n}\right\} \subset K$ such that $z_{n} \rightarrow z$ in $L^{2}(Q)$ and $y_{n} \rightarrow y$ in $L^{2}(Q)$, where $y_{n}$ (resp. $y$ ) is the solution of (3.23) associated to $z_{n}$ (resp. $y$ ). Then, we can extract a subsequence $\left\{z_{n^{\prime}}\right\} \subset\left\{z_{n}\right\}$ such that

$$
z_{n^{\prime}} \rightarrow z \text { weakly-* in } L^{\infty}(Q) .
$$

From the classical regularity estimates for the linear heat equation, we have:

$$
y_{n^{\prime}} \rightarrow y \text { weakly in } Y_{\varepsilon}:=L^{2}\left(\varepsilon, T ; H^{2}(\Omega)\right) \cap H^{1}\left(\varepsilon, T ; L^{2}(\Omega)\right)
$$

for any small $\varepsilon>0$. Observing that $Y_{\varepsilon}$ is compactly imbedded in the space $L^{2}\left(\varepsilon, T ; H_{0}^{1}(\Omega)\right)$ and $z_{n} y_{n, x}$ is uniformly bounded in $L^{2}(Q)$, we deduce that $z_{n^{\prime}} y_{n^{\prime}, x}$ converges weakly in $L^{2}(Q)$ to $z y_{x}$. Consequently, $y$ satisfies system (3.23). Now, it is readily seen that $y \in \mathcal{A}(z)$.

This ends the proof.

\subsection{Other more realistic nonlinear equations and systems}

There are a lot of more realistic nonlinear equations and systems from mechanics that can also be considered in this context. First, we have the well known Navier-Stokes equations:

$$
\begin{cases}y_{t}+(y \cdot \nabla) y-\Delta y+\nabla p=v 1_{\omega}, \quad \nabla \cdot y=0, & (x, t) \in Q \\ y=0, & (x, t) \in \Sigma \\ y(x, 0)=y^{0}(x), & x \in \Omega .\end{cases}
$$

Here and below, $Q$ and $\Sigma$ stand for the sets

$$
Q=\Omega \times(0, T) \text { and } \Sigma=\partial \Omega \times(0, T),
$$

respectively. 
To our knowledge, the best results concerning the controllability of this system have been given in [11] and [12]. The main ideas come from [15, 19]. Essentially, these results establish the local exact controllability of the solutions of (3.24) to uncontrolled trajectories (this is, more or less, the analog of the positive controllability result in theorem 3.3).

Similar results have been given in [16] for the Boussinesq equations

$$
\left\{\begin{array}{l}
y_{t}+(y \cdot \nabla) y-\Delta y+\nabla p=\theta k+v 1_{\omega}, \quad \nabla \cdot y=0, \\
\theta_{t}+y \cdot \nabla \theta-\Delta \theta=u 1_{\omega},
\end{array}\right.
$$

complemented with initial and Dirichlet boundary conditions for $y$ and $\theta$ (see [12] for a controllability result with a reduced number of scalar controls).

Another system is considered in [9]:

$$
\left\{\begin{array}{l}
y_{t}+(y \cdot \nabla) y-\Delta y+\nabla p=\nabla \times w+v 1_{\omega}, \quad \nabla \cdot y=0 \\
w_{t}+(y \cdot \nabla) w-\Delta w-\nabla(\nabla \cdot w)=\nabla \times y+u 1_{\omega} .
\end{array}\right.
$$

These equations govern the behavior of a micropolar fluid, see [24]. As usual, y and $p$ stand for the velocity field and pressure and $w$ is the microscopic velocity of rotation of the fluid particles. Again, the local exact controllability of the solutions to the trajectories is established.

Let us also mention $[2,17]$, where the controllability of the MHD and other related equations has been analyzed.

Frequently, the proof of the controllability results for these systems follows the steps of the proof of the first part of theorem 3.3. This is the scheme:

- First, consider a linearized similar problem and the associated adjoint system and rewrite the original controllability problem in terms of a fixed point equation.

- Then, prove a global Carleman inequality and an observability estimate for the latter. This provides a controllability result for the linearized problem.

- Prove appropriate estimates for the control and the state (this needs some kind of smallness of the data); prove an appropriate compactness property of the state and deduce that there exists at least one fixed point.

An alternative method is furnished by the implicit function theorem. This corresponds to a general strategy introduced in [14]:

- First, rewrite the original controllability problem as a nonlinear equation in a space of admissible "state-control" pairs.

- Then, prove an appropriate global Carleman inequality and a regularity result and deduce that the linearized equation possesses at least one solution. Again, this provides a controllability result for a related linear problem. 
- Check that the hypotheses of a suitable implicit function theorem are satisfied and deduce a local result.

At present, no negative result is known to hold for these nonlinear systems (apart from the one-dimensional Burgers equation).

\subsection{Some remarks on the Ginzburg-Landau equation}

We end this paper with a brief section devoted to the controllability of the Ginzburg-Landau equation. The system under consideration is the following:

$$
\begin{cases}m_{t}-\alpha m \times m_{t}-\Delta m+\frac{|m|^{2}-1}{\varepsilon} m=v 1_{\omega}, & (x, t) \in Q, \\ \frac{\partial m}{\partial n}=0, & (x, t) \in \Sigma, \\ m(0)=m^{0}, & x \in \Omega .\end{cases}
$$

Here, $\Omega \subset \mathbb{R}^{3}$ is a regular bounded open set, $m=\left(m_{1}, m_{2}, m_{3}\right)$ is the magnetization field, $\varepsilon>0$ is a parameter, $\alpha \geq 0$ is a physical constant and it is assumed that $m^{0}$ is a measurable initial field satisfying $\left|m^{0}\right| \equiv 1$.

In this framework, the interesting controllability problem is the following:

Given a stationary solution $m^{*}=m^{*}(x)$ and an initial field $m^{0}=$ $m^{0}(x)$ with $\left|m^{0}\right| \equiv 1$, find a control $v \in L^{2}(\omega \times(0, T))^{3}$ and an associated solution of (3.27) such that

$$
m(T)=m^{*} \text { in } \Omega .
$$

By introducing the new variable $y$, with $m=m^{*}+y$, this can be rewritten in terms of a null controllability problem. Indeed, let us consider the system

$$
\begin{cases}y_{t}-\alpha\left(y+m^{*}\right) \times y_{t}-\Delta y+G_{\varepsilon}(x, y) y=v 1_{\omega}, & (x, t) \in Q, \\ \frac{\partial y}{\partial n}=0, & (x, t) \in \Sigma, \\ y(0)=m^{0}(x)-m^{*}(x), & x \in \Omega,\end{cases}
$$

where

$$
G_{\varepsilon}(x, y) y \equiv \frac{\left|m^{*}(x)+y\right|^{2}-1}{\varepsilon}\left(m^{*}(x)+y\right)-\frac{\left|m^{*}(x)\right|^{2}-1}{\varepsilon} m^{*}(x) .
$$

Then the problem is:

For any given $m^{*}=m^{*}(x)$ and an initial field $m^{0}$ with $\left|m^{0}\right| \equiv 1$, find a control $v \in L^{2}(\omega \times(0, T))^{3}$ and an associated solution of (3.28) such that

$$
y(T)=0 \text { in } \Omega .
$$


For the moment, we can give answers to this problem only when $\alpha=0$; this will appear in [5]. It is reasonable to expect similar (positive) controllability results when $\alpha$ is sufficiently small, but at present this is an open question.

Remark 3.3 For any fixed $v$, the solutions of (3.27) can be shown to converge in some sense as $\varepsilon \rightarrow 0$ to a solution of the so called Landau-Lifshitz equation, see for instance [4]:

$$
\alpha m_{t}=m \times\left(\Delta m-m_{t}+v 1_{\omega}\right), \quad|m|=1 .
$$

Consequently, it would be very interesting to be able to solve the previous problem with controls $v$ uniformly bounded with respect to $\varepsilon$. However, this is apparently a difficult question.

Acknowledgements: The author has been partially supported by D.G.I. (Spain), Grant BFM2003-06446.

\section{References}

[1] S. Anita, D. TATARU, Null controllability for the dissipative semilinear heat equation, Appl. Math. Optim. 46 (2002), 97-105.

[2] V. Barbu, T. Havarneanu, C. Popa, S. S. Sritharan, Exact controllability for the magnetohydrodynamic equations, Comm. Pure Appl. Math. 56 (2003), 732-783.

[3] C. Bardos, G. Lebeau, J. Rauch, Sharp sufficient conditions for the observation, control and stabilization of waves from the boundary, SIAM J. Cont. Optim., 30 (1992), 1024-1065.

[4] M. Bertsch, P. Podio-Guidugli, V. Valente, On the dynamics of deformable ferromagnets. I. Global weak solutions for soft ferromagnets at rest, Ann. Mat. Pura Appl., 179 (2001), 331-360.

[5] J. L. Boldrini, E. Fernandez-Cara, S. Guerrero, Some controllability results for the Ginzburg-Landau equation, in preparation.

[6] C. Fabre, J.-P. Puel, E. Zuazua, Approximate controllability of the semilinear heat equation, Proc. Royal Soc. Edinburgh, 125 A (1995), 3161.

[7] E. Fernandez-Cara, S. Guerrero, Remarks on the null controllability of the Burgers equation, C. R. Acad. Sci. Paris, Ser. I 341 (2005), 229-232.

[8] E. Fernandez-Cara, S. Guerrero, On the null controllability of the Burgers equation, in preparation.

[9] E. Fernandez-Cara, S. Guerrero, Local exact controllability of micropolar fluids, to appear in J. Math. Fluid Mech. 
[10] E. Fernandez-Cara, S. Guerrero, Global Carleman inequalities for parabolic systems and applications to controllability, to appear in SIAM J. Control Optim.

[11] E. Fernandez-Cara, S. Guerrero, O. Yu. Imanuvilov, J.-P. Puel, Local exact controllability to the trajectories of the Navier-Stokes equations, J. Math. Pures Appl. 83, 12 (2004), p. 1501-1542.

[12] E. Fernandez-Cara, S. Guerrero, O. Yu. Imanuvilov, J.-P. Puel, Some controllability results for the $N$-dimensional Navier-Stokes and Boussinesq systems with $N-1$ scalar controls, to appear in SIAM J. Control and Optim.

[13] E. Fernández-CARA, E. ZuAZuA, The cost of approximate controllability for heat equations: The linear case, Advances Diff. Eqs., Vol. 5 (4-6), AprilJune 2000, p. 465-514.

[14] A. V. Fursikov, O. Yu. Imanuvilov, Controllability of Evolution Equations, Lecture Notes \#34, Seoul National University, Korea, 1996.

[15] A. V. Fursikov, O. Yu. Imanuvilov, Exact controllability of the Navier-Stokes and Boussinesq equations (Russian), Uspekhi Mat. Nauk 54, no. 3(327), 93-146; translation in Russian Math. Surveys 54 (1999), no. 3, $565-618$.

[16] S. Guerrero, Local exact controllability to the trajectories of the Boussinesq system, Annales IHP, Anal. non linéaire, 23 (2006), 29-61.

[17] T. Havarneanu, C. Popa, S.S. Sritharan, Exact internal controllability for the magnetohydrodynamic equations in multi-connected domains, to appear.

[18] O. Yu. Imanuvilov, Boundary controllability of parabolic equations, Russian Acad. Sci. Sb. Math., 186 (1995), 109-132 (in Russian).

[19] O. Yu. Imanuvilov, Remarks on exact controllability for the NavierStokes equations, ESAIM Control Optim. Calc. Var. 6 (2001), 39-72.

[20] O. A. Ladyzenskaya, V. A. Solonnikov, N. N. Uraltzeva, Linear and Quasilinear Equations of Parabolic Type, Trans. Math. Monograph: Moscow, 1967; Vol. 23.

[21] G. Lebeau and L. Robbiano, Contrôle exact de l'équation de la chaleur, Comm. P.D.E., 20 (1995), 335-356.

[22] J.-L. Lions, Exact controllability, stabilizability and perturbations for distributed systems, SIAM Review, 30 (1988), 1-68.

[23] J.-L. Lions, Contrôlabilité Exacte, Stabilisation et Perturbations de Systèmes Distribués, Tomes 1 \& 2, Masson, RMA 8 \& 9, Paris 1988. 
[24] G. Lukaszewicz, Micropolar fluids. Theory and applications. Modeling and Simulation in Science, Engineering and Technology, Birkhäuser Boston, Inc., Boston, MA, 1999.

[25] D.L. Russell, A unified boundary controllability theory for hyperbolic and parabolic partial differential equations, Studies in Appl. Math. 52 (1973), 189-221.

[26] D. L. Russell, Controllability and stabilizability theory for linear partial differential equations. Recent progress and open questions, SIAM Review 20 (1978), 639-739.

[27] E. ZuAzUA, Exact boundary controllability for the semilinear wave equation, in "Nonlinear Partial Differential Equations and their Applications", Vol. X (p. 357-391), H. Brezis and J.L. Lions eds., Pitman, New York 1991.

\section{Unique Continuation for Stochastic Partial Differential Equations and Its Applications}

Xu Zhang

School of Mathematics, Sichuan University, China; and

Dep. de Matemáticas, Facultad de Ciencias, Universidad Autónoma de

Madrid, Spain

xu. zhang@uam.es

\subsection{Introduction}

Unique continuation property is an important problem not only in partial differential equations itself, but also in some application problems such as controllability, optimal control, inverse problems and so on. Numerous studies on unique continuation for deterministic partial differential equations can be found in [1] and the rich references cited therein. It would be quite interesting to extend the deterministic unique continuation theorems to the stochastic ones, but there are many things which remain to be done, and some of which seem to be challenging.

There are two classical tools in the study of the unique continuation for deterministic partial differential equations. One is Holmgren-type uniqueness theorem, another is Carleman-type estimate. Note however that the solution of a stochastic equation is generally non-analytic in time even if the coefficients of the equation are constants. Therefore, one cannot expect a Holmgrentype uniqueness theorem for the unique continuation for stochastic equations unless some very special cases. On the other hand, the usual approach to employ Carleman-type estimate for the unique continuation needs to localize the problem. The difficulty for the stochastic situation consists in the fact that one cannot simply localize the problem as usual because the usual localization 